\title{
La innovación y sus implicaciones en las empresas del sector metalmecánico en la ciudad de Cartagena: caso Asimecar*
}

\section{Innovation and its implications for metallurgical sector companies in the city of Cartagena: Asimecar case study}

\author{
Nelson Eduardo Cottiz Montoya ${ }^{1}$ \\ ${ }^{1}$ Ingeniero Químico, Magíster en Administración, Fundación Universitaria Tecnológico Comfenalco. \\ ncottiz@tecnocomfenalco.edu.co
}

* Estudio de Caso resultado del trabajo de tesis para optar por el título de Magister, financiado con recursos propios, desarrollado por el autor principal desde la Unab de Bucaramanga y Tecnológico Monterrey México, en el periodo comprendido de 2010 a 2011.

Recibido: 15/feb/2021 Revisado: 30/abr/2021

Aceptado: 30/may/2021 Publicado: 30/jul/2021

\begin{abstract}
Resumen En este trabajo se analiza la capacidad de innovación de las empresas metalmecánicas de la ciudad de Cartagena (Asimecar) entre los años 2010 y 2011. Para esto, se evalúan los factores: tipos de innovación, beneficios de las innovaciones, fuentes de innovación y dificultades presentadas al momento de la implementación de la innovación. La muestra objeto de estudio estuvo constituida por todas las empresas que conforman la asociación (en total son ocho empresas). Esta investigación es de tipo descriptivo, cuantitativo, de datos primarios y los resultados muestran que las empresas metalmecánicas implementaron innovaciones organizacionales y de procesos, las cuales mejoraron la calidad de los productos, redujeron costos, entre otros aspectos. La principal fuente de innovación fueron los clientes y las principales dificultades al momento de innovar fueron la falta de recursos económicos y la falta de preparación de los empleados.
\end{abstract}

Palabras clave innovación; clases de innovaciones; fuentes de innovación; dificultades al innovar.

\begin{abstract}
In this work analyze the innovativeness of the metalworking companies of the city of Cartagena Asimecar between 2010 and 2011. For this, analyzes, factors are evaluated: types of innovation, benefits of innovation, innovation sources and difficulties faced by time of implementation of innovation. In this research, the study sample was defined by all companies that make up the association (in total there are 8 companies). This research is descriptive, quantitative primary data type. The results show that the metalworking companies implemented organizational innovations and processes, which improved product quality, cost reduction, among many. The main source of innovation was customers and major difficulties when innovation was the lack of financial resources and lack of training of employees.
\end{abstract}

Keywords innovation; kinds of innovations; innovation sources; difficulties innovate.

Teknos Revista Científica. | Volumen 21 No.1 - diciembre 2021 | ISSN 1900-7388 (papel) | ISSN 2539-2190 (digital)

DOI: https://doi.org/10.25044/issn.2539-2190 


\section{Introducción}

Nuevas economías emergentes como la India y la China, han promovido la redefinición de los negocios a nivel mundial. Estos acontecimientos han cambiado el rumbo de las empresas, ya que ahora tienen que ser más competitivas, porque la competencia no solo se da a nivel regional y nacional, sino también internacional; así, para poder establecerse y sobrevivir en el mercado, necesitan adoptar la innovación como estrategia central, con el fin de alcanzar un alto grado de competitividad.

Esta nueva redefinición del mercado ha hecho que los consumidores sean más exigentes y que tengan aún más criterios para tomar decisiones de productos y servicios. La época actual en que vivimos les exige a las empresas estar más preparadas, medirse con buenos competidores, para ser mejores y mostrar resultados en productos, procesos, diseños $y$ servicios.

Existen tres indicadores internacionales de competitividad: el índice de competitividad global, preparado por el Foro Económico Mundial (FEM); el índice de competitividad del Institute for Management Development (IMD) de Suiza y el índice de facilidad para hacer negocios (Doing Business) del Banco Mundial. En el primer indicador, Colombia se ubica en el puesto 69 entre 131 países (WEF, 2011); en el segundo, se halla en la posición 41 entre 55 países (IMD, 2011); y en el tercer indicador, se encuentra en el puesto 66 entre 131 países (DB, 2011).

Además de estos indicadores, la tendencia de Colombia en el ranking de competitividad mostrada por el Foro Económico Mundial ha decaído en los últimos tres años, tal como lo muestra la Figura 1.

Estos resultados anteriormente descritos, muestran que Colombia tiene una brecha muy grande por mejorar en su competitividad. Gran parte de esta fisura se debe a la baja productividad de la economía nacional, tanto es así que el crecimiento del producto interno bruto en Colombia en el año 2007 fue de 6,9\% y en el año 2008, fue de 3,5\%, tal como lo muestra la Figura 2. Esta disminución en la productividad colombiana es consecuencia de la carencia de procesos dinámicos de investigación, generación, gestión de conocimiento y aprendizaje, baja inversión en tecnologías de la información y telecomunicaciones, en otras palabras, falta de innovación.

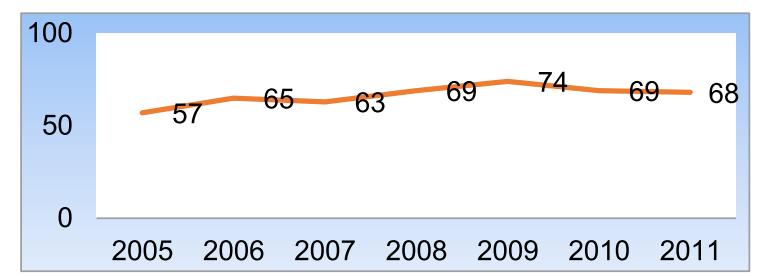

Figura 1. Posición de Colombia Ranking de CompetitividadWEF.

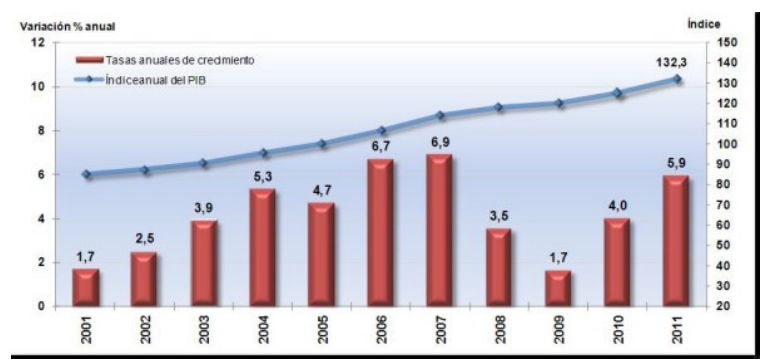

Figura 2. Producto Interno Bruto de Colombia.

Estudios realizados por Interlat, Telmex, Cisco y Fundes Colombia en el año 2008 sobre uso de internet, nuevas tecnologías y competitividad de las pymes, evidenció que el conocimiento de las pymes colombianas sobre mercadeo electrónico, negocios digitales y posicionamiento web es muy bajo, así mismo advierten que aproximadamente el $40 \%$ de las pymes nacionales tienen portal o página web donde se encuentra información corporativa relacionada con sus productos y servicios, pero no tienen ni realizan comercio electrónico ni ventas en línea. El uso de internet de las pymes se basa en consultas de correo electrónico, acceso a páginas web, intercambios de archivos y chat.

También se encontró en el estudio que las pymes colombianas invierten muy poco en competitividad. Datos arrojados muestran que solo el $30 \%$ de las empresas invierten en las TIC (Tecnologías de Información y Comunicación), el 10\% invierten en el desarrollo de nuevos productos y el $9 \%$, en el 
mejoramiento de su productividad. Además, el estudio evidenció que la gestión comercial y la estrategia de mercadeo de la pyme colombiana son nulas. Estos datos muestran un rezago tecnológico que se refleja en la baja capacidad de innovación, la cual afecta la competitividad y productividad de las pymes.

Por otro lado, un estudio realizado por el Grupo Regional de Investigación en Economía y Competitividad del Observatorio del Caribe Colombiano y el Grupo de Investigación en Economía Regional, Organización Industrial y Desarrollo Empresarial de la Cámara de Comercio de Cartagena en el año 2008, enfocado a evaluar factores de competitividad, entre ellos, el de empresas innovadoras, determinó en este indicador, que Cartagena se ubicaba en el puesto quince entre 22 ciudades de Colombia. Lo anterior dejó entrever a los investigadores que las empresas cartageneras presentan grandes falencias para desempeñarse con éxito en un contexto globalizado, debido a que no son lo suficientemente competitivas. La razón de lo anterior se debe a la falta de conocimiento en el campo innovativo, que se traduce en la no incorporación de procesos innovadores y desarrollos tecnológicos eficaces, los cuales, al no ser debidamente gestionados, generan la disminución del valor agregado en la economía; así, provocan la disminución de la productividad en la región.

Teniendo en cuenta los hechos descritos anteriormente y valorando mucho más el papel que desempeñan las pymes en nuestra nación y en nuestra región -son las principales activistas del desarrollo y del emerger económico del país-, es necesario enfocarse en la realización de la gestión de la innovación, ya que es el vínculo para que las empresas sean competitivas, para que así puedan garantizar su supervivencia en el mercado globalizado en el que vivimos.

Este trabajo se centra en definir la capacidad de innovación de las pymes que hacen parte de la Asociación de Industrias Metalmecánicas de Cartagena (Asimecar). Para esto, se evaluarán los siguientes factores: clases o tipos de innovaciones y los beneficios de esta para la empresa, fuentes de innovación y las dificultades al innovar.
Autores concuerdan que la competitividad de una empresa o región, está ligada a la creatividad, la invención y la innovación, tal como lo señala Varela (2008), quien califica a cada una de ellas como importantes en el progreso tecnológico y competitivo de toda organización, pero, además recalca que ellas presentan diferencias marcadas.

Varela define la creatividad como «la capacidad de lograr generar una idea útil y original». Por su parte, la invención «se relaciona con el desarrollo y con la materialización de una idea creativa, universalmente nueva en un prototipo, en un modelo, en un concepto, en una idea» (p. 263). Y la innovación "es el proceso mediante el cual esos prototipos o modelos, 0 conceptos o ideas, se integran al mercado y se ofrecen como bienes para ser adquiridos por los clientes» ( $p$. 263).

Igualmente, Porter (1990), citado por Escorsa y Valls (2001), destaca la innovación como un elemento esencial en la competitividad, cuando afirma que «la competitividad de una nación depende de la capacidad de su industria para innovar y mejorar. Las empresas consiguen ventajas competitivas mediante innovaciones» (p. 19). En ese mismo orden de ideas, Chesnais (1986), citado por Escorsa y Valls (2001), reitera la importancia de la innovación en el progreso de las naciones; asegura que «la actividad innovadora constituye efectivamente, con el capital humano, uno de los principales factores que determinan las ventajas comparativas de las economías industriales avanzadas» (p. 19).

Como vemos, estos autores establecen relación directa entre la competitividad y la innovación. Esta última es concebida como la culminación de un proceso creativo que desemboca en la introducción de algo nuevo en el mercado y es allí donde se centra este estudio, en la capacidad de innovación de las pymes del sector metalmecánico de Cartagena.

La innovación presenta muchos axiomas, tal es el caso de Valdés (2004), quien la explica como la capacidad de convertir una idea en un producto o servicio nuevo, novedoso y original, que tenga gran impacto en el mercado.

Piatier (1987), citado por Escorsa y Valls (2001) define la innovación como «una idea transformada en 
algo vendido o usado» (p. 18). Por su parte, Gee (1981) señala que «la innovación es el proceso en el cual a partir de una idea, invención o reconocimiento de una necesidad, se desarrolla un producto, técnica - servicio útil hasta que sea comercialmente aceptado» (Escorsa y Valls, 2001, p. 18). Como vemos, la innovación parte de una idea y finaliza con un nuevo producto o servicio que impacta al mercado.

Por otro lado, la innovación la relacionan con un proceso de cambio, como lo refiere Escorsa y Valls (2001), «la innovación es sinónimo de cambio. la empresa innovadora es la que cambia, evoluciona, hace cosas nuevas, ofrece nuevos productos $y$ adopta, o pone a punto, nuevos procesos de fabricación» (p. 15). También Drucker (1996), citado en Varela (2008), señala que la innovación es «la búsqueda organizada y sistemática, con un objetivo de cambio, de las oportunidades que existan en el ambiente» (p. 267).

Hasta el momento, los autores han esquematizado la innovación como un proceso de cambio, que parte de una idea y que genera un nuevo producto o servicio. Pero, otros autores van más allá, enmarcan la innovación no solo como lo definieron anteriormente, sino que agregan otros conceptos, tal es el caso de Schumpeter (1934), citado en Escorsa y Valls (2001), quien definió la innovación en los siguientes casos:

- La introducción en el mercado de un nuevo bien, un bien con el cual los clientes no están aún familiarizados.

- La introducción de un nuevo método de producción, un método no probado en el sector industrial; también existe innovación en una nueva forma de comercializar un nuevo producto.

- La apertura de un nuevo mercado en un país.

- La conquista de una nueva fuente de suministro de materias primas o de productos semielaborados.

- La importación de una nueva estructura en un mercado.

La innovación comprende más que el desarrollo de un nuevo producto o servicio y cambio permanente en la organización, también abarca el desarrollo de nuevas técnicas organizacionales, nuevas formas de comercializar un producto o servicio. De una forma más general, se puede definir la innovación como: "la introducción de un nuevo, o significativamente mejorado producto (bien o servicio), de un proceso, de un nuevo método de comercialización o de un nuevo método organizativo, en las practicas internas de la empresa, la organización del lugar de trabajo o las relaciones exteriores" (OCDE, 2005, p. 56).

La innovación puede ser clasificada de diferentes formas. Valdés (2004) las clasifica según su impacto en el mercado. Entre estas, tenemos: (a) las innovaciones graduales o evolutivas, (b) la innovación de rompimiento y (c) la arquitectura de nuevos modelos de negocio.

El primero corresponde a un cambio mínimo en el producto, el cual es percibido y valorado por el mercado. Esta innovación busca mejorar el rendimiento del producto 0 servicio original, modificando favorablemente la percepción que el usuario tiene del producto o servicio.

La innovación gradual, aunque parezca sorprendente para el mercado, no modifica dramáticamente el producto o servicio original, ni representa un salto tecnológico cualitativo importante, tampoco representa grandes barreras contra el copiado, ya que tienden a ser imitadas y superadas por la competencia.

Por su parte, las innovaciones de rompimiento se generan cuando hay un importante avance tecnológico o se crea un nuevo concepto que modifica el mapa de competencia. Esta clase de innovaciones generan un nivel superior de competencias $y$ modifican radicalmente la percepción del cliente. Estas mejoras producen grandes saltos cualitativos y radicales respecto al producto o servicio original.

Tales innovaciones se pueden dividir en dos grupos: las de rompimiento tecnológico y las conceptuales. Las de rompimiento tecnológico son aquellas donde se presenta una tecnología superior que vuelve obsoleta la anterior y desarrolla un salto cualitativo y cuantitativo en el producto o servicio original. $Y$ las innovaciones conceptuales se refieren al desarrollo de un nuevo producto o servicio, más amplio y diferente, que mejora drásticamente las funciones del producto o servicio anterior. 
A este último tipo de innovaciones se le conoce como aplicaciones asesinas, ya que al aparecer en el mercado y ser aceptadas por el cliente, vuelven obsoletas las aplicaciones anteriores. Las diferencias entre estas radican en que en la innovación de rompimiento tecnológico se dan grandes saltos dentro del mismo producto o servicio, mientras que en la innovación conceptual, se generan cambios en la esencia de la idea original del producto o servicio.

Por último, la arquitectura de nuevos modelos de negocios, se refiere a un tipo de innovación que se enfoca al modo cómo una empresa diseña su negocio para poder competir en el mercado.

Mejorar radicalmente la manera cómo se hacen los negocios es la manera de competir, así lo sustenta Hamel (2000), citado por Valdés (2004): «cada negocio no debería estar enfocado a mejorar lo que funcionó ayer, sino en producir formas nuevas y revolucionarias de hacer los negocios del mañana» ( $p$. 154).

Las empresas que modifican sustancialmente su sector industrial y crean un nivel de competencia superior, lo hacen gracias a la habilidad de reinventarse a sí mismas, al desarrollo de nuevos conceptos de negocios innovadores, al cambio radical de la esencia del negocio; buscan, principalmente, abrir otros mercados, servir a nuevos clientes, desarrollar nuevas fuentes de ventajas competitivas, generar fuentes de ingresos diferentes e ingresar al mercado de una forma única y diferenciada.

Otra manera útil de clasificar las innovaciones es la siguiente: principales (o radicales) e incrementales, tal como lo señala Escorsa et al. (2001). Las innovaciones «principales o radicales suponen una rotura súbita respecto al estado anterior» (p. 29). Estas innovaciones se ven reflejadas en mejoras espectaculares en los productos o servicios, mas no en la reducción de costos. Por su parte, las innovaciones incrementales son concebidas por las mejoras de los productos o procesos ya conocidos. Esta clase de innovación se manifiesta en la reducción de costos, los cuales se plasman en la curva de aprendizaje o de experiencia.

Por otra parte, el Manual de Oslo (OCDE, 2005) clasifica las innovaciones en: innovaciones de producto, innovaciones de proceso, innovaciones de mercadotecnia e innovaciones de organización. La innovación de producto: [...] corresponde con la introducción de un bien o de un servicio nuevo, o significativamente mejorado, en cuanto a sus características o en cuanto al uso al que se destina. Esta definición incluye la mejora significativa de las características técnicas, de los componentes y los materiales, de la informática integrada, de la facilidad de uso u otras características funcionales (p. 58).

La innovación de proceso representa «la introducción de un nuevo, o significativamente mejorado, proceso de producción o de distribución. Ello implica cambios significativos en las técnicas, los materiales y/o los programas informáticos» (OCDE, 2005 , p. 59). Por su parte, la innovación de mercadotecnia contempla «la aplicación de un nuevo método de comercialización que implique cambios significativos del diseño o el envasado de un producto, su posicionamiento, su promoción o su tarificación» ( $p$. 60). Esta innovación también se conoce como innovación de comercialización. Por último, la innovación organizacional se define como «la introducción de un nuevo método organizativo en las prácticas, la organización del lugar de trabajo o las relaciones exteriores de la empresa» (p. 62).

Ahora bien, las innovaciones en la empresa de alguna manera tributan en un beneficio. Desde este punto de vista, el Manual de Oslo (OCDE, 2005) establece que las innovaciones de producto y de procesos generan beneficios en la empresa, tales como: gama más amplia de bienes o servicios, penetración en nuevos mercados, mayor participación de mercado, mejor calidad de bienes o servicios, más flexibilidad en la producción o la prestación de servicios, mayor capacidad de producción o prestación de servicios, menores costos, cumplimiento de normas, menor impacto ambiental.

Por su parte, las innovaciones de mercadotecnia crean una mayor participación en el mercado, una introducción de productos a nuevos mercados o nuevos grupos de clientes, un aumento de la visibilidad de los productos o negocios, una mejora de la capacidad para responder a las necesidades del cliente. 
Y las innovaciones organizativas apuntan a: la reducción del periodo de respuesta a las necesidades del cliente o proveedor, mayor calidad de bienes o servicios, menores costos, mejora de la satisfacción del personal, mejora del intercambio de información dentro de la empresa.

En el proceso de gestión de la innovación es muy importante determinar las fuentes de innovación. Ante esto, Valdés (2004) propone las siguientes: innovación orientada por las necesidades percibidas de los clientes, innovación orientada por las necesidades no percibidas de los clientes y la innovación orientada por las necesidades futuras de los clientes; las cuales son orientadas y diseñadas por el cliente, con el fin de entender sus necesidades, requisitos y deseos.

Con respecto a la primera, el autor postula que, para alcanzar el éxito, es necesario orientar la innovación, donde se busque lograr un nuevo valor agregado a las necesidades del cliente. Las necesidades percibidas del cliente son las que él sabe que tiene, es consciente o semiconsciente de ellas y es capaz de expresarlo. Las necesidades especificas de un cliente pueden dar lugar al desarrollo de una innovación, por lo que el conocimiento de estas necesidades son claves para el desarrollo del proceso innovativo.

El conocimiento de las necesidades se puede obtener mediante diferentes técnicas; entre ellas se encuentran: las cualitativas, las cuales evalúan los deseos del cliente, y las cuantitativas, que miden el nivel de requerimiento y la intensidad de cada una de ellas. En la vida real se encuentran muchas técnicas y mecanismos que arrojan información importante para desarrollar el perfil de necesidades del cliente. Esta clase de necesidades percibidas de los clientes no desarrollan una ventaja competitiva a largo plazo, debido a que muchas empresas están enfocadas a satisfacerlas.

En las necesidades no percibidas por los clientes, Valdés (2004) establece que son más reveladoras e interesantes. Las técnicas cualitativas y cuantitativas no son útiles para recolectar información, debido a que el cliente no es consciente de sus necesidades, por el contrario, la información indirecta que se extrae de los estudios de los estilos y formas de vida del consumidor, se convierte en una fuente confiable.

Nicolai (2002), citado por Valdés (2004) afirma que según estudios realizados de diferentes hábitos $\mathrm{y}$ estilos de vida, «una persona pasa por seis etapas en su vida adulta, vinculadas con su economía, forma de vida y hábitos de compra» (p. 81). Cada una de estas etapas presentan necesidades específicas y las innovaciones deben estar enfocadas a cubrirlas. Las seis etapas son: soltero mayor de 18 años, casado sin hijos, casado con hijos menores de 12 años, casado con hijos mayores de 12 años, nido vacío (los hijos ya no viven con ellos), soltero (uno de los cónyuges falleció o están divorciados).

Por otro lado, la innovación orientada por las necesidades futuras de los clientes, está ligada a los avances tecnológicos que se van desarrollando en el mundo de la ciencia. Esta clase de productos y servicios innovativos aparecen de tal modo que el cliente no se imagina que puedan llegar a existir. La tecnología se ha convertido en parte fundamental de nuestras vidas, gracias a la gran erupción que ha tenido, nos han llevado a la generación de nuevos materiales, sistemas, productos, diseños y elementos que han producido cambios en la percepción del consumidor y en los gustos del cliente. En cuanto a empresas, los avances tecnológicos las han ayudado a la reducción de costos, disponibilidad, capacidad de respuesta, etc. Estas innovaciones tecnológicas se manifiestan a través de un ciclo de vida que se divide en cuatro etapas según Sterling (2002), citado por Valdés (2004): interrogación, estrella naciente, vaca lechera y perro muerto.

Es importante que las organizaciones estén al tanto de los avances tecnológicos. Por ello, Valdés (2004) resalta las fuentes que sirven para monitorearlos: Universidades y centros de investigación, revistas especializadas en tecnología, revistas especializadas en innovación, revistas especializadas en gerencia, foros especializados, congresos y seminarios, redes de especialistas, sitios de Internet.

Los empresarios siempre han estado al tanto de los cambios que se producen en el mercado. Se mantienen alerta y en plena comunicación abierta con los proveedores y clientes, asisten a ferias técnicas, 
foros, etc. Pero, en los tiempos actuales, es muy difícil detectar a tiempo lo que está sucediendo, ya que gran parte de la información presenta un crecimiento exponencial y circula a través de los «colegios invisibles», los cuales son grupos de expertos, profesionales 0 académicos de diferentes países que se comunican entre sí. Ante esta situación, Escorsa et al. (2001) proponen diferentes criterios o enfoques, dependiendo del área en la cual la empresa desea estar informada, con el fin de vigilar las posibles fuentes de innovación:

- A partir de los aportes de Porter (1990), citado por Escorsa y Valls (2001), se determinaron los cuatro factores de competitividad de las empresas: clientes, proveedores, entrantes potenciales en el mercado y productos substitutos; estos son ejes claves que la empresa debe vigilar.

- A partir de los factores críticos de éxito de Rockart (Jakobiak, 1991, citado por Escorsa y Valls, 2001), que son «aquellos aspectos críticos de los que depende la buena marcha de la empresa. Estos factores son inherentes al sector de la actividad, dependen de los objetivos y la estrategia de la empresa y son variables en el tiempo» (p. 76).

Otra manera para determinar y clasificar las fuentes de innovación la describe Afuach (1999), citado por Arraut (2007). Afuach clasifica las fuentes de innovación en funcional y circunstancial. La funcional tiene que ver con la procedencia de las innovaciones, sean externas o internas. De esta clase existen cinco fuentes de innovación:

- Cadena de valores internos. Cualquier departamento de la empresa puede proveer fuentes de innovación.

- Cadena externa de valores agregados de proveedores, clientes e innovadores complementarios.

- Laboratorios universitarios, gubernamen-tales y privados.

- Competidores e industrias relacionadas.

- Otras naciones y regiones.

Por su parte, las fuentes de innovación circunstancial contemplan cuándo 0 en qué circunstancias es de esperarse que ocurra la innovación. Estas se clasifican en:

- Actividades planificadas de la organización.

- Ocurrencias inesperadas.

- Cambio, destrucción creativa.

Las innovaciones no llegan todas a ser exitosas, algunas generan impactos dañinos dentro de la organización y otras impactan negativamente al mercado, debido a que en el proceso innovativo, se encuentran obstáculos o factores que entorpecen esta labor. Ante esta situación, el Manual de Oslo (OCDE, 2005) describe cuatro factores:

a. Factores de costos. Estos se dan por la falta de fondos financieros, ya sea porque la organización no tiene los suficientes recursos para financiar la innovación o porque la innovación resulta tener un costo muy elevado.

b. Factores de conocimiento. Estos se presentan por la ausencia de personal calificado, falta de información sobre tecnología, falta de información sobre los mercados, dificultades para encontrar socios de cooperación para la innovación.

c. Factores de mercado. Se presentan cuando hay incertidumbre respecto a la demanda de bienes o servicios o cuando los mercados son dominados por empresas establecidas.

d. Factores motivacionales. Se muestran cuando en la organización, las innovaciones anteriores no permiten que se genere nuevas innovaciones.

Por su parte, Valdés (2004) propone tres obstáculos que limitan la innovación en el mercado, estos son: los empleados, la comunicación con el cliente y la sociedad. El autor señala que los empleados, parte fundamental de la empresa y los principales gestores del cambio, son los primeros en rechazar este proceso, puesto que tienen la concepción errónea que el cambio les generará la pérdida de sus puestos de trabajo. Otro factor que puede entorpecer la innovación es la comunicación, ya que esta puede ser deficiente, debido a que lo que quiere comunicar la empresa no es en realidad lo que entiende el cliente. Por último, el autor señala que la sociedad puede no aceptar la innovación porque no 
está preparada o porque malinterpreta el mensaje de la empresa.

Ahora bien, se han llevado a cabo muchos estudios en innovación. Entre estos, se destaca el realizado por Malaver y Vargas (2002-2003), quienes investigaron la relación que hay entre la competitividad y la tecnología con el surgimiento y desarrollo de los procesos de innovación. En el estudio, se analizaron las variables: fuentes de innovación, procesos de innovación, clases de innovación, efectos e impactos de las innovaciones. Los resultados mostraron que las innovaciones que se efectuaron fueron de producto y de procesos, las cuales se originaron en: fuentes internas, tales como el gerente, departamento de investigación y desarrollo, departamento de producción y ventas; y en fuentes externas, tales como clientes, proveedores, consultores y universidades. Los efectos e impactos de las innovaciones incidieron en la parte económica, incrementando las ventas y el empleo; en la parte competitiva, se aumentaron los márgenes, la cuota de mercado y el desarrollo de competencias tecnológicas; y en la parte tecnológica, se acrecentó la capacidad de hacer proyectos más complejos, se evidenció una actitud positiva frente a la tecnología y aumentó la frecuencia de las innovaciones.

También, tenemos el estudio realizado en la industria turística por Jacob y Bravo (2001). Dicho estudio tuvo como fin determinar la importancia de la innovación y las clases de innovaciones en la industria turística. Entre las variables analizadas, se encuentra el número de innovaciones realizadas: fueron de 142 innovaciones entre 20 empresas encuestadas. También, evaluaron las clases de innovaciones, donde las de proceso y comercialización fueron las más desarrolladas. Las fuentes donde provinieron las innovaciones descritas anteriormente surgieron principalmente de la dirección y los clientes. Otra variable examinada fue la motivación para innovar, donde la mayoría de las empresas innovan con el fin de sobrevivir, hacerlo mejor y ganar dinero. Por otra parte, las dificultades que arrojó el estudio al momento de innovar se debieron por falta de personal, resistencia interna y falta de financiación. El beneficio logrado por la innovación se reflejó en la imagen, rentabilidad y satisfacción del cliente.
Calderón y Naranjo (2007) en su estudio dirigido a empresas metalmecánicas de la ciudad de Manizales, encontró que estas se caracterizaron por ser fuertes en la generación de nuevos productos, en la mejora significativa de productos, procesos y servicios y en la gestión del conocimiento en busca de nuevas ideas. Por otra parte, Sánchez (2008) determinó en su investigación que las principales dificultades al innovar de las empresas metalmecánicas de la ciudad de Risaralda obedecieron a la carencia de talento humano calificado, a la cultura organizacional y a la falta de compromiso con la innovación.

El observatorio de Navarro, por su parte, desarrolló un estudio de innovación en las empresas, en el que analizó los tipos de innovación, las dificultades de la innovación y sus causas y efectos. Los resultados demostraron que durante el periodo 2005 al 2007, se introdujeron innovaciones de producto, de procesos, de comercialización y de organización; las innovaciones de producto y de proceso fueron las que en mayor número se lograron, según la información de las empresas encuestadas. Las principales dificultades de la innovación obedecieron a los costos elevados, a la falta de información del mercado, falta de información de la tecnología y falta de personal calificado. Entre los beneficios generados por la innovación de producto se destaca: mayor gama de mercancías, mayor cuota de mercado y la introducción en nuevos mercados. Así, la innovación de procesos originó beneficios en la reducción de materiales, reducción de costos e incremento de la producción.

\section{Materiales y métodos}

Para desarrollar esta investigación, se desarrolló un instrumento que estaba dividido en cinco partes. La primera correspondía a los datos de la empresa a entrevistar. La segunda parte hacía referencia a indagar las clases de innovación ejecutadas en la empresa. La tercera correspondía a los beneficios alcanzados por la empresa al implementar una innovación. El siguiente fragmento del cuestionario contempló las fuentes de innovación. La última sección estuvo dirigida a determinar las dificultades de 
las empresas al momento de desarrollar e implementar la innovación.

Dicho instrumento fue contestado en una entrevista personal por cada uno de los gerentes de las empresas que conforman la Asociación de Industrias Metalmecánicas de Cartagena (Asimecar).

Las empresas que están vinculadas a esta asociación son: Bustos Compañía Ltda., Metal Prest, Técnica Metalmecánica del Caribe, Ferretería Taller Ignacio Sierra, Industrias Fervill Ltda., Fumeco, Servitec e Imec. Para este trabajo, se realizó una investigación descriptiva, dado que nos permite detallar los tipos de innovación, beneficios al innovar, fuentes de innovación y dificultades al innovar de las empresas pertenecientes a Asimecar. Igualmente, se utilizó análisis formal y estructurado, apoyado por métodos estadísticos para el razonamiento de los resultados.

\section{Resultados}

\subsection{Tipos de innovaciones y sus beneficios}

En este trabajo de la gestión innovativa de las empresas que hacen parte de la Asociación de Industrias Metalmecánicas de Cartagena (Asimecar), una de las variables que se evaluó fue: tipos de innovaciones y beneficios alcanzados en el periodo 2010 - 2011.

Para determinar los tipos de innovaciones que alcanzaron las empresas del sector, se tuvieron en cuenta principalmente los conceptos sobre innovación de producto, innovación de procesos, innovación de comercialización e innovación organizativa, que se encuentran referenciados en el Manual de Oslo (OCDE, 2005). Para ello, al entrevistado se le aplicó un cuestionario estructurado con trece preguntas, cada una apuntaba a indagar cuál de los cuatro tipos de innovaciones habían alcanzado (innovación de producto, innovación de proceso, innovación organizativa e innovación de comercialización).

De acuerdo con los resultados de las entrevistas, se evidenció que el $87,5 \%$ de las empresas alcanzaron algún tipo de innovación durante el periodo 2010 -
2011 (el 87,5\% corresponde a siete empresas de ocho). Por el contrario, el $12,5 \%$ de las empresas no alcanzaron ningún tipo de innovación (el 12,5\% corresponde a una empresa de ocho).

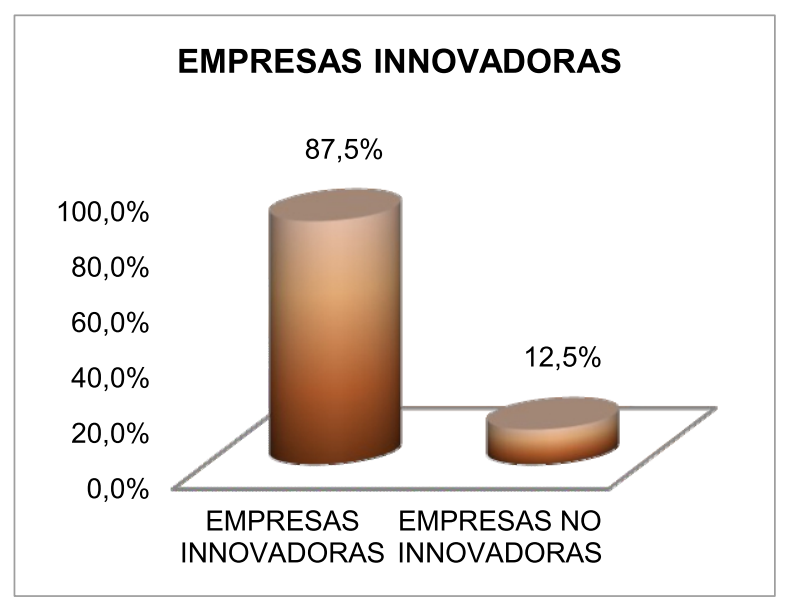

Figura 4. Empresas innovadoras.

Estos resultados sobresalientes se deben al apoyo y formación recibida por parte de la Cámara de Comercio de Cartagena, Sena, Confecámaras y Acopi, quienes con su esfuerzo, han desarrollado una red de colaboración con el fin de apoyar a las empresas pertenecientes a la Asociación de Industrias Metalmecánicas de Cartagena (Asimecar), mediante iniciativas como el Programa Digital Mipymes Caribe, un proyecto financiado por el Ministerio de Comunicaciones para orientar, capacitar y fomentar el uso de las Tecnologías de Información y Comunicaciones (TIC) en las micro, pequeñas y medianas empresas de la región Caribe, con el objetivo de disminuir la brecha tecnológica existente y así, aumentar la competitividad y la productividad.

Asimismo, se desarrolló el proyecto «Diseño e Implementación del Sistema de Gestión de la Calidad ISO 9001 para las empresas del sector Metalmecánico de Cartagena», el cual logró la certificación de trece empresas metalmecánicas bajo esta norma. Igualmente, se destaca el proyecto patrocinado por Cardique, Sena, Alcaldía de Cartagena y Universidad Tecnológica de Bolívar denominado Programa de Excelencia Ambiental (Prea). Dicho proyecto estuvo dirigido a las empresas metalmecánicas de Cartagena 
con el objetivo de contribuir, capacitar y acompañar al mejoramiento continuo del desempeño ambiental; además, promovió en este sector productivo, la adopción de tecnologías y comportamientos que privilegian la prevención como estrategia principal de preservación ambiental y estimuló la implementación de prácticas en las empresas para mejorar su gestión ambiental.

Por último, destacamos los seminarios y cursos realizados por Acopi, Bolívar y por la Universidad Tecnológica de Bolívar a las empresas pertenecientes a la Asociación de Industrias Metalmecánicas de Cartagena (Asimecar), de los cuales se destacan: Innovación y Creatividad y el Seminario Taller Herramientas de Gestión de la Innovación. Este último tuvo como objetivo conocer la importancia estratégica que tiene la innovación para la competitividad de las empresas y dar a conocer herramientas de gestión de la innovación.



Figura 5. Clases de innovaciones.

Estos esfuerzos descritos anteriormente se han visto reflejados también en el número de empresas que han implementado innovaciones; vemos que el $13 \%$ de ellas implementaron innovaciones de producto, el $63 \%$ de las empresas desarrollaron innovaciones de procesos y el $75 \%$ efectuaron innovaciones organizativas. Como observamos en la Figura 5, la mayoría de las empresas introdujeron innovaciones de procesos, lo que se debió a la implementación de nuevas técnicas y a la implementación de nuevos equipos automatizados (equipos robotizados); esto apuntaba a la necesidad imperiosa de las empresas por disminuir sus costos y por mejorar la calidad de sus productos.

Las innovaciones organizativas fueron también las más implementadas por las empresas de la Asociación de Industrias Metalmecánicas de Cartagena (Asimecar); lo anterior se debió a la introducción de nuevos métodos para organizar las rutinas y los procedimientos de gestión de los trabajos. También, reorganizaron los lugares de trabajo mediante la integración de las distintas actividades laborales dentro de la empresa; por ejemplo, efectuaron la producción sobre pedido, el cual integra las ventas y la producción.

Cabe destacar el hecho de que ninguna empresa de la Asociación de Industrias Metalmecánicas de Cartagena (Asimecar) implementó innovaciones de comercialización, ya que prácticamente las compañías enfocaron todo su esfuerzo a la disminución de sus costos, a la mejora de la calidad de los productos y a la mejora de la organización.

Tabla 1. Innovaciones por empresa

\begin{tabular}{|l|l|l|l|}
\hline \multicolumn{1}{|c|}{ EMPRESA } & $\begin{array}{c}\text { I. } \\
\text { PRODUCTO }\end{array}$ & $\begin{array}{c}\text { I. } \\
\text { PROCESOS }\end{array}$ & $\begin{array}{c}\text { I. } \\
\text { ORGANIZATIVA }\end{array}$ \\
\hline $\begin{array}{l}\text { Bustos Compañia } \\
\text { Ltda. }\end{array}$ & & & \\
\hline Metal Prest & & & \\
\hline $\begin{array}{l}\text { Técnica } \\
\text { Metalmecánica del } \\
\text { Caribe }\end{array}$ & & & \\
\hline $\begin{array}{l}\text { Ferretería Taller } \\
\text { Ignacio Sierra }\end{array}$ & & & \\
\hline $\begin{array}{l}\text { Industrias Fervill } \\
\text { Ltda. }\end{array}$ & & & \\
\hline Fumeco & & & \\
\hline Servitec & & & \\
\hline Imec & & & \\
\hline
\end{tabular}

En la tabla 1, se detallan las empresas y las clases de innovaciones que alcanzaron. Como se ilustra en la tabla, la empresa Bustos Compañía Ltda., implementó innovaciones de producto, de procesos y organizativa. Las empresas Metal Prest, Técnica Metalmecánica del Caribe y Fumeco, lograron implementar innovaciones 
de procesos y organizativas. Las metalmecánicas Ferretería Taller Ignacio Sierra y Servitec implementaron innovaciones organizativas y la empresa IMEC implemento innovación de procesos. Todo lo contrario paso con la empresa Fervill Ltda., quien no alcanzó ningún tipo de innovación en el periodo comprendido del 2010 al 2011 , debido a que la empresa, no ha desplegado un liderazgo que apoye la innovación y además, se ha acostumbrado y conformado con sus actividades internas que no han tenido la necesidad de innovar para cambiar la manera en que funciona y opera la empresa, lo cual ha desarrollado varios factores que entorpecen aún más el inicio de un proceso innovativo, como son la falta de asignación de recursos económicos que conlleve a la mejora de las actividades internas de la empresa, la ausencia de herramientas y de procesos que estimulen y motiven al empleado a generar ideas que conlleven a la innovación, y por último, la ausencia en la misión, de una estrategia definida que describa, oriente y guie a la empresa mediante la utilización e implementación de la innovación a ser más competitivos y a aumentar el nivel de satisfacción del cliente.

Estas innovaciones alcanzadas deben impactar o beneficiar de alguna forma a las empresas. Por ello, se investigó el impacto o el beneficio alcanzado mediante un cuestionario aplicado al entrevistado, el cual constó de doce preguntas (pregunta 18 - 29 del cuestionario). Estas preguntas tenían calificaciones que variaban de uno a cinco, donde uno significaba «no importante»; dos, «poco importante»; tres, «algo importante»; cuatro, «importante» y cinco significaba «muy importante».

En la Figura 6 se presentan los beneficios alcanzados por las empresas que implementaron algún tipo de innovación. Como se evidencia en ella, la mayoría de las compañías alcanzaron beneficios en: mayor calidad de bienes o servicios, mayor flexibilidad en la producción o prestación de servicios, menos costos, menor impacto ambiental, mejora del intercambio de información dentro de la empresa, mayor capacidad de producción o prestación de servicios, reducción del periodo de respuesta al cliente o proveedor y lealtad al cliente.
Estos beneficios que representan el mayor puntaje por parte de las empresas concuerdan con los citados en el Manual de Oslo (OCDE, 2005), el cual afirma que, si se presentan innovaciones de procesos, estos deben impactar en la reducción de los costos, mejorar la calidad de los productos y permitir una mayor flexibilidad en la producción. Por otro lado, el Manual de Oslo sostiene también que, si se presentan innovaciones organizativas, estas deben reducir el periodo de respuesta al cliente y mejorar el intercambio de información dentro de la empresa. Dichos beneficios concuerdan con los resultados mostrados por las empresas que integran la Asociación de Industrias Metalmecánicas de Cartagena (Asimecar).

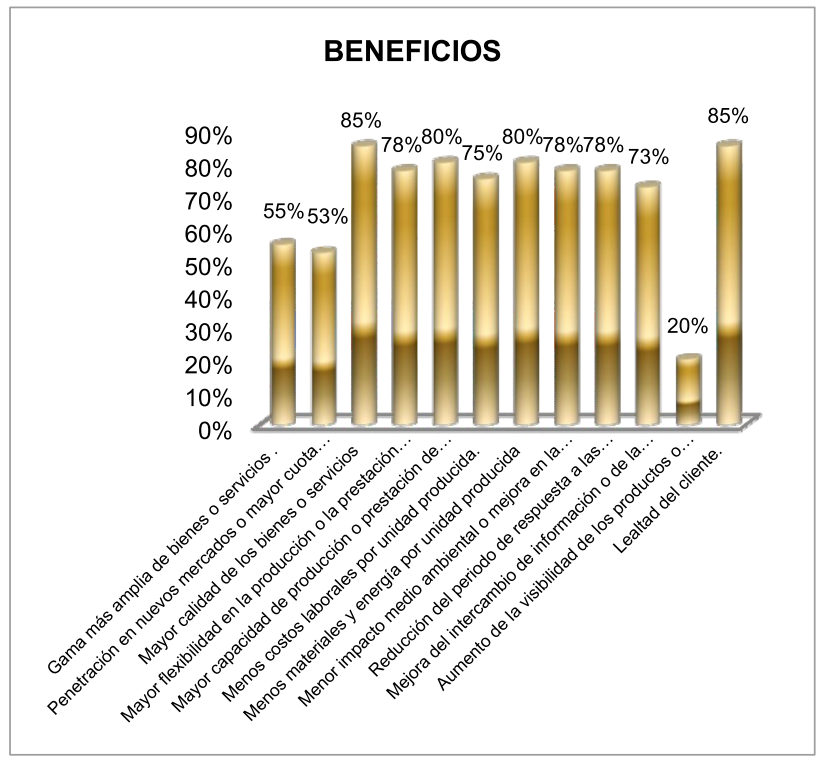

Figura 6. Beneficios al innovar.

Hay que destacar que existen beneficios que de una u otra manera son consecuencias de las mejoras que implementan las empresas, ya que, si la organización innova de manera efectiva, dispone de clientes más leales y se aumenta la cuota de negocios con ellos. También los empleados se benefician, porque consiguen mayores y mejores resultados; consiguen una acentuación de orgullo y compromiso por pertenecer a la empresa. 


\subsection{Fuentes de innovación}

Otra variable que se analizó en esta investigación fue la procedencia de las ideas que generaron la innovación o la mejora, en otras palabras, las fuentes de innovación. Para medir esta variable, a los entrevistados, se les formularon once interrogantes. Estas preguntas debían ser calificadas de uno a cinco, donde uno significaba «no importante»; dos, «poco importante»; tres, «algo importante»; cuatro, «importante» y cinco significaba «muy importante».

En la Figura 7 se detallan las principales fuentes que dieron origen a la actividad innovadora. Como se muestra, vemos que los clientes, la información dentro de la empresa y las universidades $u$ otros centros de enseñanza superior, obtuvieron los mayores puntajes.

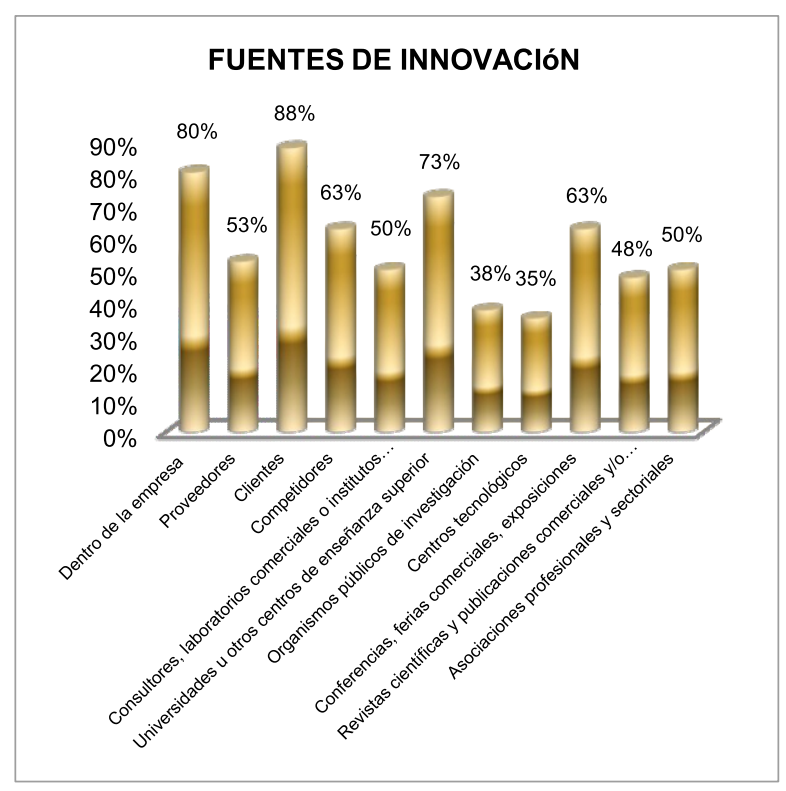

Figura 7. Fuentes de Innovación.

Para las empresas, los clientes son su principal y fundamental fuente de innovación, debido a que tienen contacto directo y constante con ellos y mantienen una buena comunicación, mediante encuestas de satisfacción o por contacto directo con sus propios empleados. Lo anterior les ha permitido conocer la opinión de lo que siente y quiere el cliente y que a su vez, este sugiera ideas de mejora para el producto o servicio prestado, algo muy productivo para la empresa para anticipar las tendencias y en definitiva, para el futuro del sector. A través de esta estrategia, han conseguido un sinnúmero de ideas provenientes de clientes que los ha ayudado a innovar.

Otra fuente de innovación que obtuvo un puntaje importante fue la información dentro de la empresa. Este resultado concuerda con el informe de innovación de la IBM denominado Expanding the innovation horizon: The Global CEO Study 2006, el cual sostiene que la fuente más importante de ideas innovadoras en las empresas son los empleados, puesto que estos trabajan en contacto con los clientes, tienen a cargo proyectos y mantienen comunicación con el resto de las personas de la compañía. Esta situación les genera un conocimiento sobre todas las situaciones laborales que se puedan mejorar desde el puesto de trabajo de cada uno de ellos.

Las universidades, instituciones con responsabilidad social que tiene como finalidad responder a las necesidades de formación de las personas y generar conocimiento, son otras de las principales fuentes de innovación de las empresas que integran la Asociación de Industrias Metalmecánicas de Cartagena (Asimecar). Lo anterior se debe a las buenas relaciones entre la asociación y la Universidad Tecnológica de Bolívar. Dicha institución se ha caracterizado por ser una gestora del conocimiento científico que alimenta el proceso innovador y ayuda a la transferencia de nuevas tecnologías: presta consultorías, cooperación y asistencia técnica a la industria, especialmente a las empresas metalmecánicas de Cartagena.

Por otra parte, las fuentes de innovación menos utilizadas por la asociación fueron: los competidores, las conferencias, las ferias comerciales, las exposiciones, los proveedores, los consultores, las revistas científicas y las asociaciones profesionales y sectoriales. Como vemos, todas estas fuentes de innovación que son mínimamente utilizadas son externas a las empresas que integran la Asociación de Industrias Metalmecánicas de Cartagena (Asimecar). Lo anterior corrobora la idea propuesta por Chesbrough, quien afirma que muchas empresas practican un modelo obsoleto de innovación que se conoce como innovación cerrada y se fundamenta en 
que el flujo de ideas que permite la innovación proviene del capital intelectual al interior de la empresa; a diferencia de la innovación abierta u open innovation, el cual Chesbrough lo definió como un modelo innovativo que fomenta la generación de ideas y de tecnologías dentro y fuera de la empresa con la participación de los competidores, organizaciones públicas y privadas, proveedores, etc.

\subsection{Dificultades al innovar}

En el presente trabajo, también se analizó la variable correspondiente a las dificultades encontradas al momento de innovar. El Manual de Oslo (OCDE, 2005) describe cuatro factores que impiden un proceso innovativo exitoso, ellos son: factores de costos, factores de conocimiento, factores de mercado y factores motivacionales. Para averiguar la incidencia de dichos factores, se le pidió al entrevistado que contestara un cuestionario conformado por once preguntas (interrogantes enumerados del 41 al 51 del cuestionario). Estas preguntas debían ser calificadas de uno a cinco, donde uno significaba «no importante»; dos, «poco importante»; tres, «algo importante»; cuatro, «importante» y cinco significaba «muy importante».

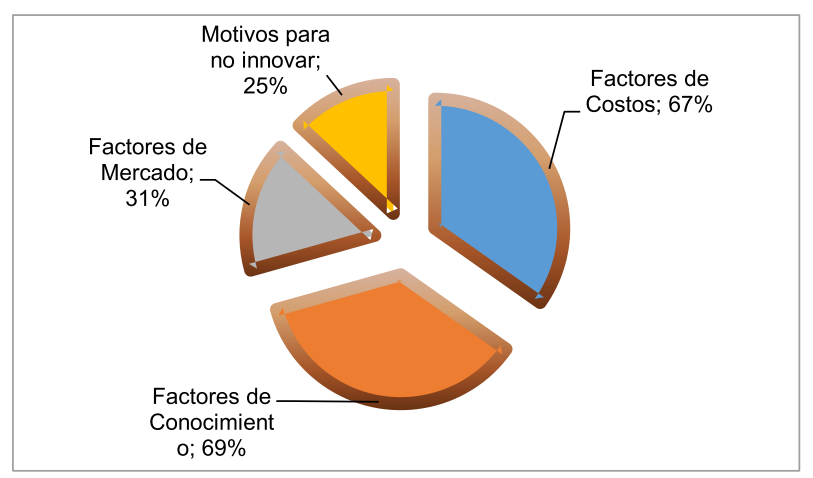

Figura 8. Dificultades al innovar.

En la Figura 8, se presentan los resultados de las principales dificultades que obstaculizan la innovación. Como vemos, los factores de conocimiento y los factores de costos, con el $69 \%$ y el $67 \%$ respectivamente, representan los principales obstáculos que encuentran las empresas. Lo anterior se debe a que el proceso innovativo conlleva una gran cantidad de recursos financieros que muchas de las empresas integrantes de la Asociación de Industrias Metalmecánicas de Cartagena (Asimecar) no tienen.

También hay que reconocer que diversas compañías que intentaron realizar una actividad innovadora, no encontraron respaldo económico con las entidades financieras ni con empresas privadas. Además, cuando llevan a cabo una mejora, ya sea que adquirieron una nueva máquina para optimizar significativamente el proceso de producción, encuentran que el conocimiento que presentan los empleados no es el necesario o suficiente para poder desarrollar un proceso exitoso. Por tal motivo, a las empresas que han llevado a cabo actividades innovativas, donde implementan algo nuevo al interior de las empresas, les toma mucho más tiempo de lo presupuestado para que el recurso humano se acople de la mejor manera al cambio.

\section{Conclusiones}

En este trabajo, se concluye que las compañías que hacen parte de la Asociación de Empresas Metalmecánicas de Cartagena (Asimecar) desarrollaron innovaciones de tipo organizativas y de procesos en su gran mayoría. Estas innovaciones impactaron positivamente en las compañías generando reducción en costos, mejora en la calidad de los productos, optimización de los procesos de fabricación, mejora en la producción de servicios, mayor confianza del cliente y menor impacto ambiental.

Las innovaciones desarrolladas por las empresas metalmecánicas de la asociación provinieron principalmente de los clientes, al interior de la empresa y de las universidades; sin embargo, se evidenció que estás empresas no monitorean como fuente de innovación las ferias comerciales, ponencias, proveedores, consultores y revistas científicas.

Las principales dificultades que encontraron las empresas al momento de innovar se centraron en la falta de recursos financieros y en la ausencia de 
conocimientos por parte de sus empleados para gestionar el proceso innovativo.

\section{Referencias}

Arraut, Luis Carlos (2007). Elementos Claves Para Generar la Capacidad Emprendedora Para el Desarrollo de Organizaciones Innovadoras. Extraído el 10 de Diciembre de 2008 de: http://espacio.uned.es/fez/eserv/bibliuned:19358/n04arrautc0 7.pdf.

Bravo, A.; Jacob, M. (2001). Estudio exploratorio sobre innovación en el sector turístico Balear. MadridEspaña: Editorial.

Calderón, Gregorio; Naranjo, Julia (2007). Perfil cultural de las empresas innovadoras. Un estudio de casos de empresas metalmecánicas. Revista Cuadernos de Administración, 20(34), 161-189.

Doing Business (2011). Informe Anual: Doing Business 2008. Recuperado el 10 de Noviembre de 2011 de: www. doingbusiness.org.

Escorsa, Pere; Valls, Jaume (2001). Tecnología e Innovación en la Empresa. México: Alfaomega.

Cámara de Comercio de Cartagena y Observatorio del Caribe Colombiano (2008). Indicador Global de Competitividad de las Ciudades Colombianas, 2008: El caso de Cartagena de Indias. Cartagena: Observatorio del Caribe Colombiano.

IBM Corporation (2006). Expanding the innovation Horizon: The global CEO study 2006. Orlando, Florida: IBM Corporation.

Institute For Management Development (2011). Informe Anual de 2008. Recuperado el 4 de Noviembre de 2011, de www.imd.ch/index.cfm?nav1=true.

Interlat Group (2008). Uso de internet y nuevas tecnologías como herramientas para las Pymes Colombianas Exportadoras o potencialmente exportadoras.

Malaver, F.; Vargas, M. (2002-2003). Estudio de caso sobre el proceso de innovación, sus determinantes e impacto en la industria manufacturera Colombiana. Extraído el 16 de Diciembre de 2008 de: http://www.scielo.org.co/scielo.php?script=sci arttext\& pid=S0120-35922004000100003

Organización para la Cooperación y el Desarrollo Económico (OCDE) (2005). Oslo Manual: Guidelines for Collecting and Interpreting Innovation, 3rd Edition. París: OECD Publications.

Sánchez, Jhon. (2008). Obstáculos que limitan la capacidad e innovación de las empresas del sector metalmecánico de Risaralda Colombia. Pereira: Universidad Tecnológica de Pereira.

Valdés, Luigi (2004). Innovación. El Arte de Inventar el Futuro. Bogotá: Grupo Editorial Norma.

Varela, Rodrigo (2008). Innovación Empresarial: Arte y Ciencia en la creación de empresas. $3^{a}$ Edición. Santiago de Cali: Pearson.

World Economic Forum (2011). Informe Anual 2008. Recuperado el 10 de Diciembre de 2011, de www.weforum.org/en/index.htm. 